\title{
Chemical and structural analysis of sub-20 nm graphene patterns generated by scanning probe lithography
}

Arancha I. Dago ${ }^{1}$, Soraya Sangiao ${ }^{2,3}$, Rodrigo Fernández-Pacheco ${ }^{2}$, José María De Teresa ${ }^{2,3,4^{*}}$ and Ricardo Garcia ${ }^{*}$

${ }^{1}$ Materials Science Factory, Instituto de Ciencia de Materiales de Madrid (ICMM), CSIC, Sor Juana Inés de la Cruz 3, 28049 Madrid, Spain

${ }^{2}$ Laboratorio de Microscopías Avanzadas (LMA), Instituto de Nanociencia de Aragón (INA), Universidad de Zaragoza, 50018 Zaragoza, Spain

${ }^{3}$ Departamento de Física de la Materia Condensada, Facultad de Ciencias, Universidad de Zaragoza, 50009 Zaragoza, Spain

${ }^{4}$ Instituto de Ciencia de Materiales de Aragón (ICMA), Universidad de Zaragoza - CSIC, 50009 Zaragoza, Spain

\begin{abstract}
Sub-20 nm patterns have been fabricated by using oxidation scanning probe lithography on epitaxial graphene. The structural and chemical properties of these nanopatterns have been characterized by high resolution transmission electron microscopy, energy dispersive X-ray spectroscopy and electron energy loss spectroscopy. The electron microscopy images reveal that the nanolithography process modifies the graphene monolayer and a thin region of the $\mathrm{SiC}$ substrate (1 nm thick). Spatially-resolved electron spectroscopies show that the nanopatterns are made of graphene oxide. The combination of spatially-resolved structural and chemical analysis of graphene nanopatterns will enable the development of highperformance graphene devices.
\end{abstract}

*Corresponding authors. E-mail: deteresa@unizar.es (J.M. de Teresa), r.garcia@csic.es (R. Garcia) 


\section{Introduction}

Scanning probe lithography (SPL) is being used to fabricate different nanolectronic devices by modifying and/or manipulating 2D electronic materials [1-3]. Among SPL methods, oxidation scanning probe lithography (o-SPL) enables the direct and resist-less nanopatterning of a large variety of materials [4-6], from silicon to 2D electronic materials; from self-assembled monolayers to biomolecules. The direct and resist-less lithography offered by o-SPL has generated a variety of nanopatterns on layered materials such as graphene and transition metal dichalcogenides [7-12]. The patterning capability has also been exploited to fabricate several electronic devices including quantum point contacts and fieldeffect transistors on layered materials [13-20].

Oxidation SPL operates by confining laterally the local oxidation of a surface. A water bridge between the tip and the sample produces the confinement. This water bridge is induced by the application of an external voltage (figure 1(a)). The water bridge also provides oxyanions that drive the oxidation process in the presence of an applied voltage. The dielectric properties, the size and the geometry of the o-SPL patterns are the key features to confine the electron flow on the active sections of the graphene layer (figure 1(b)).

Advances in the performance of those devices require a full understanding of the structural and chemical properties of those dielectric barriers. Current knowledge on the chemical and dielectric properties of o-SPL patterns is based on experiments performed on silicon [21,22]. Recently, micro-Raman and X-ray spectroscopy have been applied to characterize submicrometer o-SPL patterns on graphene [23, 24]. However, there are no studies on the chemical and the structural properties of those nanopatterns that have the spatial resolution relevant for the development of high-performance devices. 

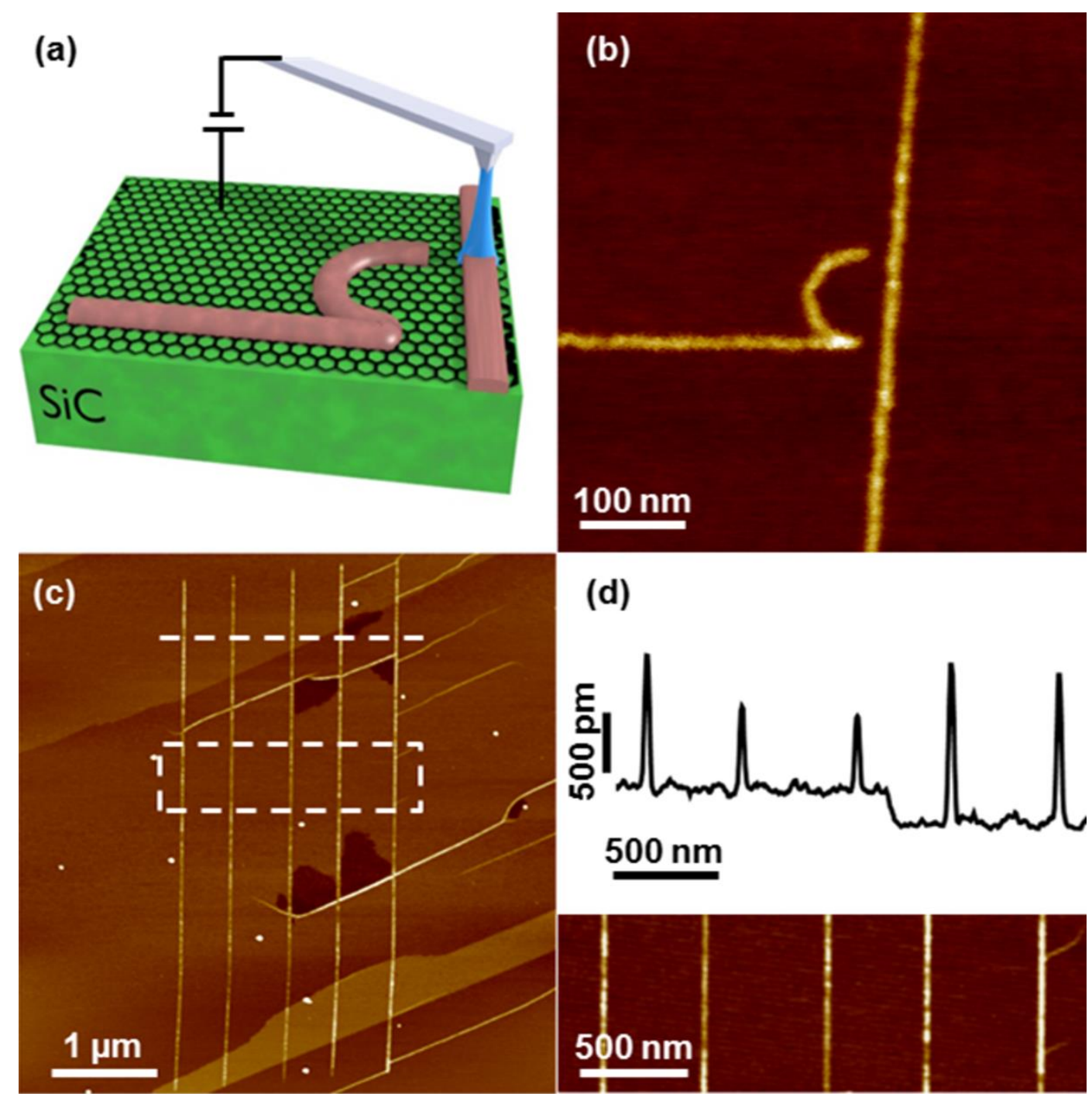

(d)

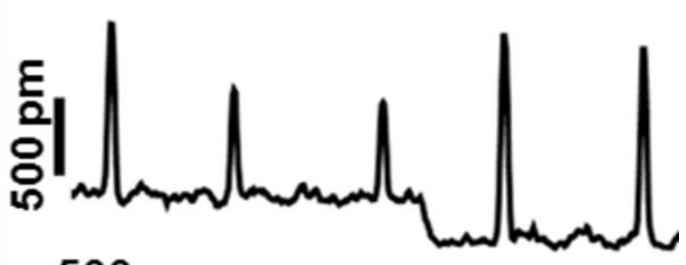

$500 \mathrm{~nm}$

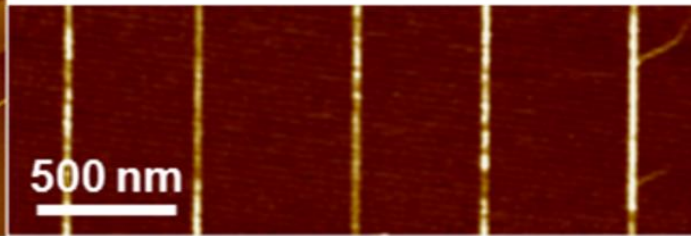

Figure 1. (a) Scheme of o-SPL patterning on a graphene layer formed on SiC. (b) o-SPL pattern on graphene. The narrowest section between the o-SPL patterns is of $18 \mathrm{~nm}$. (c) Array of lines patterned on graphene by o-SPL. (d) Top panel shows the cross-section along the dashed line marked in (c). Bottom image is a high-resolution image of the area enclosed in the rectangle marked in (c). o-SPL parameters applied voltage $V=37 \mathrm{~V}$ and oxidation time $t=1 \mathrm{~ms}$ in (b) and $V=20 \mathrm{~V}$ and $t=0.7 \mathrm{~ms}$ in (c).

Here we report a combined force microscopy, high-resolution transmission electron microscopy (HRTEM), focused ion beam nanolithography and high spatial resolution electronic spectroscopy to characterize the structure and chemical composition of nanopatterns fabricated by o-SPL on epitaxial graphene. The nanopatterns characterized in this report have a lateral width in the sub-20 nm range while their height is below $4 \mathrm{~nm}$.

Epitaxial graphene is being actively studied for its potential towards wafer-scale fabrication of graphene-based devices [25-27]. The patterning of graphene by optical or electron-beam 
lithographies requires the use of resists. Those resists might deteriorate the structural and/or electrical properties of the patterns and devices [28]. This observation supports the use of a resist-free direct lithography technique such as o-SPL to pattern epitaxial graphene. Previous results of o-SPL on epitaxial graphene have shown the potential of this technique for the patterning of graphene on $\mathrm{SiC}$ [29-31], although high-resolution nanopatterns have not been produced so far for this type of graphene. Moreover, a deep characterization of the processes involved in o-SPL of graphene with nanometer resolution is missing. In our work, the spectroscopy analysis shows that the patterns contain $\mathrm{C}$ and $\mathrm{O}$ which confirms the existence of a graphene oxide. The o-SPL patterns grow 1-3 nm above and below the graphene baseline. This also implies that the patterning affects the $\mathrm{SiC}$ underneath by the incorporation of Si into the graphene oxide. However, by tuning the oxidation parameters involved in the $\mathrm{o}-\mathrm{SPL}$ process, it is possible to limit the oxidation process to the graphene layer.

The patterns have a trapezoidal shape dominated by the width of the base. We have shown that for the smallest and thinnest oxides (total thickness of about $2 \mathrm{~nm}$ ), the shape is almost rectangular. This is important in order to define the real distance between the dielectric barriers in a quantum dot device.

\section{Experimental}

The o-SPL experiments were performed by operating the atomic force microscope (dimension V, Bruker, USA) in the amplitude modulation mode with a free amplitude in the 5-10 $\mathrm{nm}$ range and a set point amplitude/free amplitude ratio of about 0.9 [5]. The local anodic oxidation experiments were carried out by using $\mathrm{n}^{+}$-doped silicon cantilevers (NCHW, NanoWorld, Germany) with a force constant of about $40 \mathrm{~N} / \mathrm{m}$ and a resonant frequency of about $300 \mathrm{kHz}$. During the nanolithography process, the tip and the sample were kept in a closed chamber to control the relative humidity, in the range of $40 \%-50 \%$ and the temperature at approximately $25^{\circ} \mathrm{C}$. To modify the graphene, the tip was biased negatively with respect to the sample. The height and the width (FWHM) of the patterns are modulated by adjusting the amplitude and duration of the voltage pulse. We have applied voltage pulse of 20-40 V for about 0.5-2 ms. Given that the local oxidation of SiC can depend on the crystal orientation [32], all our o-SPL processes have been performed on the same substrate terrace with the oxidation lines parallel amongst them, so, along the same crystallographic direction. 
The graphene samples were produced by Graphene Nanotech on the silicon face of SiC substrates. The growth of graphene is produced through selective sublimation of Si surface atoms by high temperature annealing. The desorbed Si atoms leave behind an epitaxial layer of graphene [33].

In order to determine the morphology and atomic structure of the materials, HRTEM was performed in a Titan Cubed TEM (FEI Company) equipped with an image-aberration corrector. On the other hand, to determine the composition of the materials, ScanningTransmission Electron Microscopy (STEM) was performed in a Titan TEM (FEI Company) equipped with a probe corrector. Both microscopes were operated at a working voltage of $300 \mathrm{kV}$. TEM images were obtained with a coupled CCD camera (Gatan), whereas High Angle Annular Dark Field (STEM-HAADF) images were obtained with a HAADF detector (Fischione) in scanning-transmission mode. In this technique, the intensity of the signal is proportional to the square of the atomic number $\left(\mathrm{Z}^{2}\right)$; therefore heavier elements appear with a much brighter contrast than lighter elements. The lamellae for the cross-sectional TEM studies have been fabricated in a Helios Nanolab 650 (FEI Company). Milling and initial thinning was performed at $30 \mathrm{kV}$ acceleration voltage for the $\mathrm{Ga}$ ions and final polishing was performed using $5 \mathrm{kV}$ Ga ions. The Pt-C layer on graphene shown in the images of figure 2(b) and 2(c) is grown during the lamellae preparation before the milling step to avoid graphene damage.

Moreover, in order to analyze the chemical composition of the materials with a high spatial resolution, Energy Dispersive X-ray Spectroscopy (EDX) and Electron Energy Loss Spectroscopy (EELS) were performed in STEM mode. EDX spectra were obtained with an EDAX detector, whereas EELS spectra were obtained with a Gatan Tridiem 866 ERS filter, with an energy dispersion of $0.2 \mathrm{eV}$ per pixel. In both techniques, spectra were acquired from integrated areas in order to obtain a better signal/noise ratio. 


\section{Results and discussion}

Figure 1(a) shows a scheme of the o-SPL patterning on graphene monolayer grown on a SiC substrate. Figure 1(b) shows a pattern on graphene that confines the flow of the electrons through an $18 \mathrm{~nm}$ wide constriction. This type of patterns has been fabricated to study the electronic properties of graphene quantum dots [16].

To perform the structural and spectroscopic characterization of the nanopatterns we have patterned arrays of lines on epitaxial graphene (figure 1(c-d)). Those patterns have been processed by focused ion beam lithography to create lamellae that were suitable for structural analysis performed by HRTEM.

Figure 2(a) shows the AFM image and the cross-section of two lines that were used in the spectroscopic analysis. Figure 2(b) shows the HRTEM image of one of those lines. Highresolution images of the cross-section show the high crystallinity and order of the $\mathrm{SiC}$ substrate. It also reveals the presence of two graphene monolayers. One attached to the substrate that is defective for the electronic transport and the other one, $0.4 \mathrm{~nm}$ above it. The substrate crystallinity could be slightly altered in the regions underneath the patterns. In this case, the local oxidation process alters the graphene layer and about 1-2 $\mathrm{nm}$ of the $\mathrm{SiC}$ underneath. The lateral extension is about $17 \mathrm{~nm}$, which matches the size given by the AFM cross-section images (figure 2(a)). The images reveal that the pattern grows about and below the substrate baseline. By tuning the o-SPL parameters, this is by reducing the applied voltage below $20 \mathrm{~V}$ and the oxidation time below $0.5 \mathrm{~ms}$ the process could be confined just $1 \mathrm{~nm}$ above or below the graphene layer (figure 2(c)). 


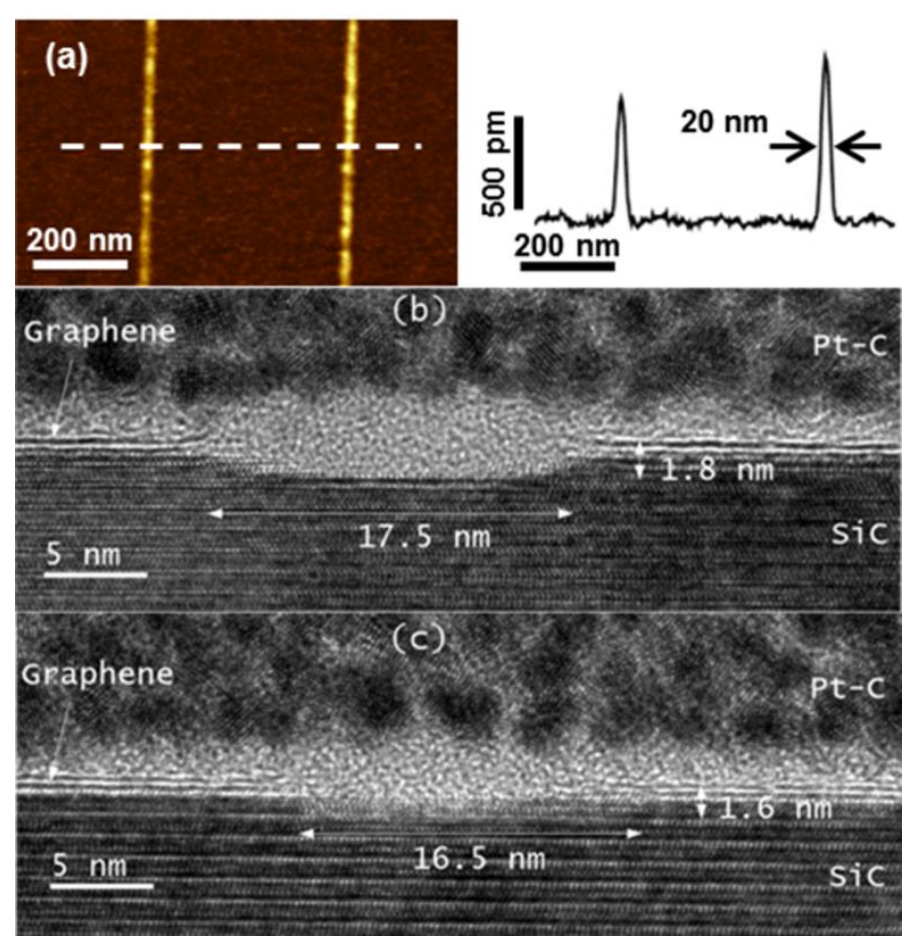

Figure 2. (a) AFM topography image of two parallel lines fabricated on graphene. The right panel shows the cross-section along the dashed line marked in the AFM image. (b) HRTEM image of a section of one of the lines of figure 2(a). (c) HRTEM image of another o-SPL line.

To study the chemical properties of the o-SPL on epitaxial graphene, EELS spectra of O K edge were acquired for different lines patterned by o-SPL. First, the exact location of the oSPL graphene line is marked during HRTEM experiments by producing a defect in the protective Pt-C layer, just above the o-SPL graphene line position. This on-purpose defect can be observed in the HAADF-STEM image shown in figure 3(a) as a bright white mark centered on the protective Pt-C layer. A spectrum of the O K edge measured in STEM mode after integration over the green-boxed area shown in figure 3(a) for an o-SPL graphene line is presented in figure 3(b). The strong signal observed for the $\mathrm{O} \mathrm{K}$ edge at the EELS spectrum, is a sign of the oxidation of graphene [34]. The O K-edge spectrum has an intense initial peak at $543 \mathrm{eV}$ and an extended broad second peak at about $566 \mathrm{eV}$, showing strong similarities with the O K-edge spectrum obtained in suspended graphene oxide flakes [35]. The O Kedge spectrum shown in figure 3(b) suggests that the graphene line patterned by o-SPL has a width of at least $35 \mathrm{~nm}$, in contrast to the value found in HRTEM images, figures 2(b) and 2(c), in which the width of the o-SPL graphene line is about $17 \mathrm{~nm}$. The reason for this discrepancy is that HRTEM is a much more reliable technique for determining spatial 
dimensions. EELS signal has less spatial resolution because it is broadened due to scattering delocalization [36]. In addition, figure 3(c) shows the integrated EELS signal over an energy window between 534 and $584 \mathrm{eV}$, corresponding to the $\mathrm{O} \mathrm{K}$ edge, as a function of the position along the red line shown in figure 3(a). The signal of the $\mathrm{O} \mathrm{K}$ edge is present along the width of the graphene oxide line, decreasing mildly near the end of the oxidized line, and disappearing completely at the non-oxidized epitaxial graphene regions. EELS spectroscopy analysis confirms the presence of graphene oxide in the area of the graphene layer that has been patterned by o-SPL and that graphene oxide is present only in the o-SPL graphene line, since the signal from the $\mathrm{O} \mathrm{K}$ edge rapidly decreases below the detection limit as we reach the non-oxidized epitaxial graphene region. 

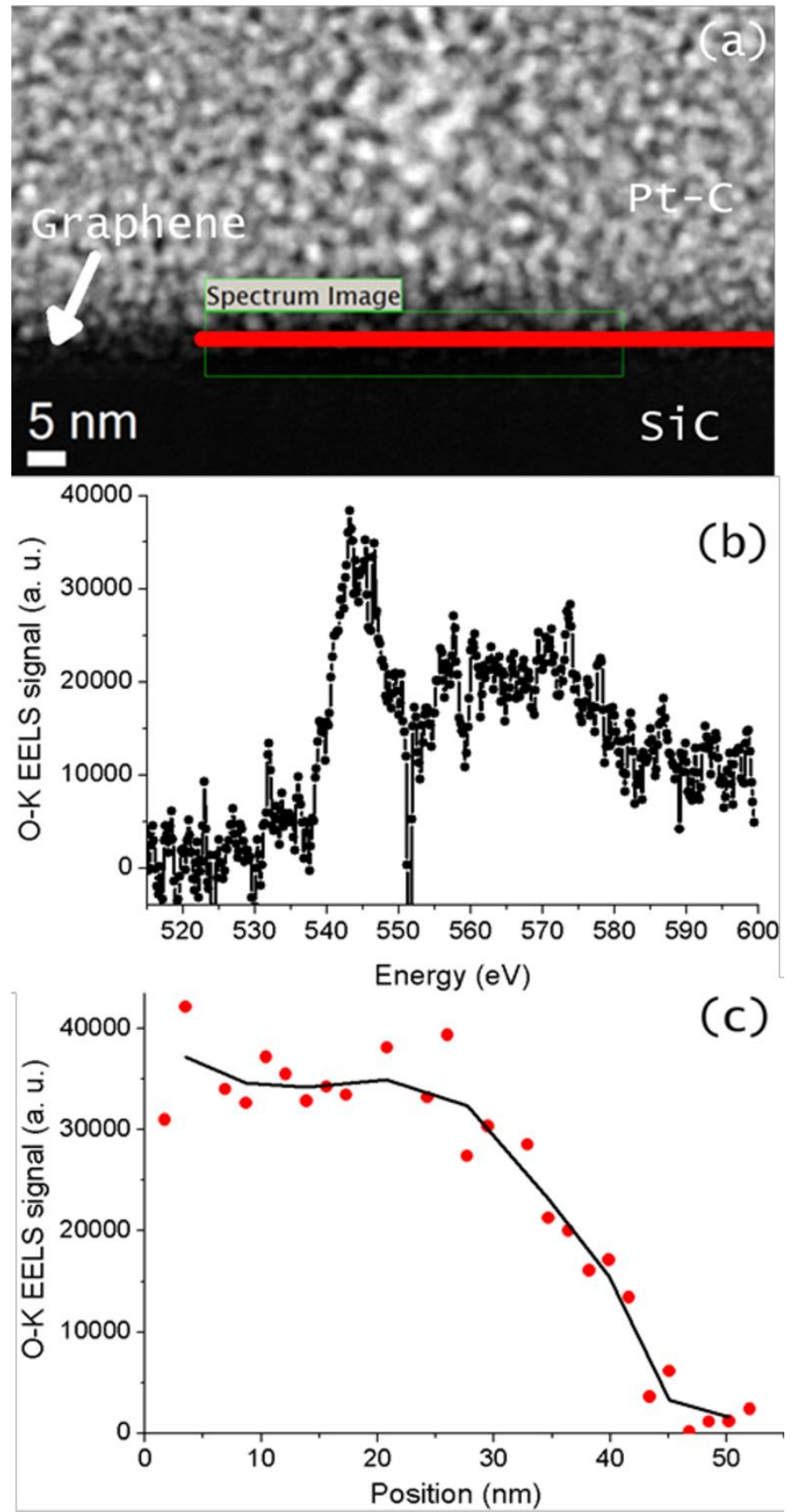

Figure 3. (a) Cross-sectional STEM image of one graphene oxide line used as a reference image for EELS and EDX analysis. Solid line is a guide to the eye. (b) Integrated EELS signal obtained after integration over the green rectangle shown in (a). (c) Integrated EELS signal over an energy window between 534 and $584 \mathrm{eV}$ corresponding to the $\mathrm{O} \mathrm{K}$ edge as a function of the position along the red line shown in (a). 
To further verify that the presence of graphene oxide is limited into the area nanopatterned by o-SPL, EDX analysis was carried out on the graphene samples. Figure 4(a) shows the HAADF-STEM image of graphene containing one graphene oxide line patterned by o-SPL (the graphene oxide line created on graphene by o-SPL is placed below the white mark created on the protective Pt-C layer, that can be seen figure 4(a). This mark has been produced in order to easily locate the graphene oxide line in subsequent STEM experiments). EDX spectra were obtained after integration over the blue and red boxed areas shown in figure 4(a), corresponding to an area where graphene oxide line is present (blue rectangle) and another area where the graphene monolayer has not been patterned by o-SPL (red rectangle). EDX spectra obtained on each area are shown in figure 4(b), with the blue line corresponding to the area containing the graphene oxide line and red line corresponding to the EDX spectrum of the unpatterned graphene monolayer. EDX spectra obtained in both areas show the presence of $\mathrm{C}$, coming from the epitaxial graphene, the $\mathrm{SiC}$ substrate and the protective $\mathrm{Pt}-\mathrm{C}$ layer, of $\mathrm{Si}$, coming from the $\mathrm{SiC}$ substrate, and $\mathrm{Pt}$, resulting from the protective Pt-C layer. Interestingly, the presence of the $\mathrm{O} \mathrm{K}$ peak is evident at the region that suffered the controlled oxidation process by o-SPL, whereas is completely absent at the region of the epitaxial graphene that suffered no modification. 


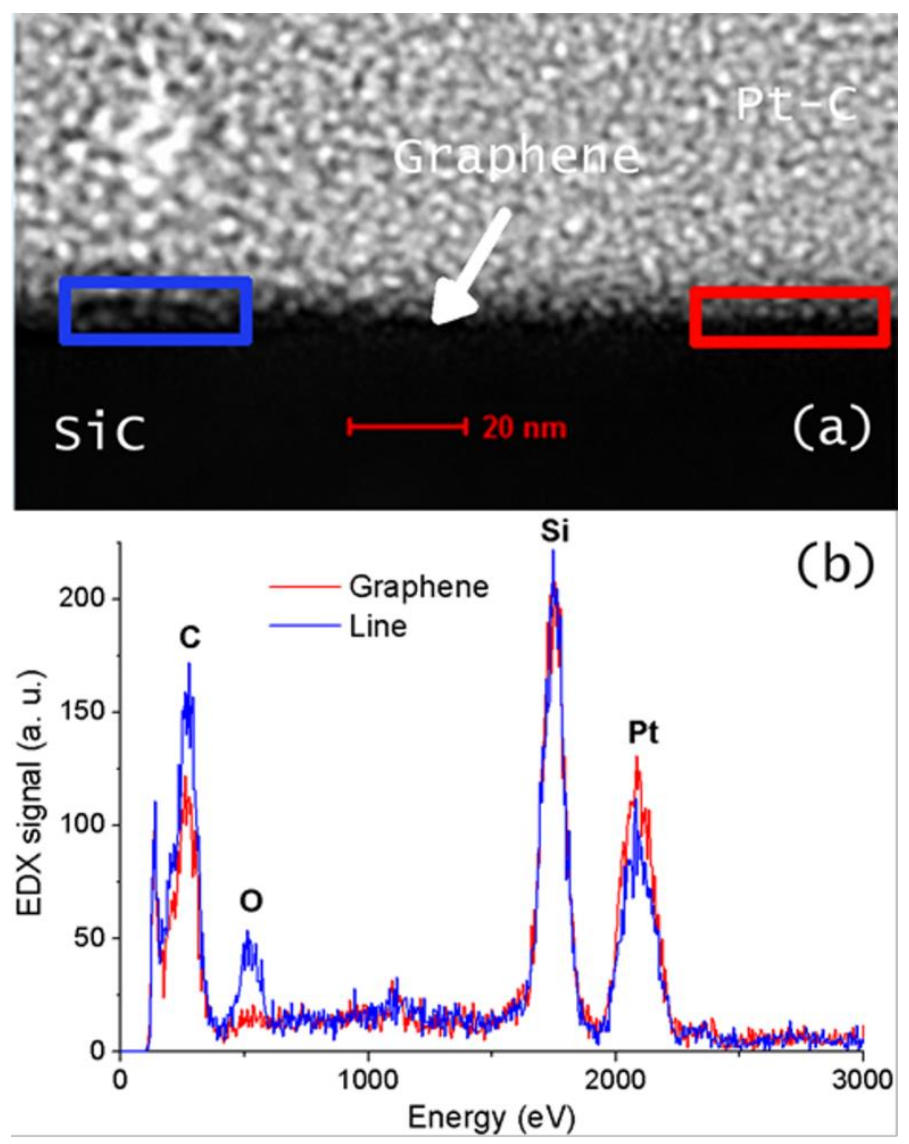

Figure 4. (a) Cross-sectional STEM image of graphene containing one graphene oxide line used as reference for EDX analysis. (b) Integrated EDX signals corresponding to the signals acquired in each rectangle shown in (a). Blue rectangle corresponds to the graphene oxide area and red rectangle correspond to a graphene region without graphene oxide lines.

\section{Conclusions}

In short, we have shown here that using o-SPL it is possible to produce high-resolution nanopatterns on epitaxial graphene. We have performed a thorough characterization of the structural and chemical properties of sub-4 nm in height and sub-20 nm in width graphene oxide patterns created by o-SPL on epitaxial graphene.

HRTEM analysis reveals that, due to the appropriate tuning of the oxidation parameters involved in the nanopatterning, o-SPL process alters the graphene layer and 1-2 $\mathrm{nm}$ of the $\mathrm{SiC}$ substrate underneath and that the lateral extension of the graphene oxide lines patterned is about $17 \mathrm{~nm}$. Spectroscopic analysis by EELS and EDX confirm the presence of graphene 
oxide along the graphene line patterned by o-SPL and the absence of any oxygen signal outside the nanopatterned areas.

These results represent the first comprehensive investigation of the structural and chemical properties of o-SPL patterns on graphene and pave the way for future research on design of high-performance graphene devices, where a precise nanopatterning is required by a resistfree direct lithography technique.

\section{Acknowledgements}

Financial support by several projects are acknowledged: MAT2014-51982-C2-1-R, MAT2014-51982-C2-2-R and MAT2015-69725-REDT, MAT2016-76507-R from MINECO (including FEDER funding) and Aragón Regional Government through project E26-Construyendo Europa desde Aragón. This work was also funded by the European Union FP7/2007-2013 under Grant Agreement No. 318804 (SNM). 


\section{References}

[1] Garcia R, Knoll AW, Riedo E. Advanced scanning probe lithography. Nat Nanotech 2014; 9(8):577-87.

[2] Kurra N, Reifenberger RG, Kulkarni GU. Nanocarbon-scanning probe microscopy synergy: Fundamental aspects to nanoscale devices. ACS Appl Mater Interfaces 2014; 6(9):6147-63.

[3] Wei Z, Wang D, Kim S, Hu Y, Yakes MK, Laracuente AR, et al. Nanoscale tunable reduction of graphene oxide for graphene electronics. Science 2010; 328(5984):1373-76.

[4] Garcia R, Martinez RV, Martinez J. Nano-chemistry and scanning probe nanolithographies. Chem Soc Rev 2006; 35(1):29-38.

[5] Liu H, Hoeppener S, Schubert US. Nanoscale materials patterning by local electrochemical lithography. Adv Eng Mater 2016; 18(6):890-902.

[6] Ryu YK, Garcia R. Advanced oxidation scanning probe lithography. Nanotechnology 2017; 28 (14):142003.

[7] Weng L, Zhang L, Chen YP, Rokhinson LP. Atomic force microscope local oxidation lithography of graphene. Appl Phys Lett 2008; 93(9):093107.

[8] Byun IS, Yoon D, Choi JS, Hwang I, Lee DH, Lee MJ, et al. Nanoscale lithography on monolayer graphene using hydrogenation and oxidation. ACS Nano 2011; 5(8):6417-24.

[9] Lorenzoni M, Giugni A, Di Fabrizio E, Perez-Murano F, Mescola A, Torre B. Nanoscale reduction of graphene oxide thin films and its characterization. Nanotechnology 2015; 26 (28):285301.

[10] Donarelli M, Perrozzi F, Bisti F, Paparella F, Feyer V, Ponzoni A, et al. Few layered $\mathrm{MoS}_{2}$ lithography with and AFM tip: description of the technique and nanospectroscopy investigations. Nanoscale 2015; 7(26):11453-59.

[11] Pinilla-Cienfuegos E, Mañas-Valero S, Navarro-Moratalla E, Tatay S, Forment-Aliaga A, Coronado E. Local oxidation nanolithography on metallic transition metal dichalcogenides surfaces. Appl Sci 2016; 6 (9): 250.

[12] Liu X, Chen K-S, Wells SA, Balla I, Zhu J, Wood JD, et al. Scanning probe nanopatterning and layer-by-layer thinning of black phosphorus. Adv Mater 2017; 29(1): 1604121. 
[13] Masubuchi S, Ono M, Hirakawa K, Machida T. Fabrication of graphene nanoribbon by local anodic oxidation lithography using atomic force microscope. Appl Phys Lett 2009; 94(8):082107.

[14] Neubeck S, Ponomarenko LA, Freitag F, Giesbers AJM, Zeitler U, Morozov SV, et al. From one electron to one hole: quasiparticle counting in graphene quantum dots determined by electrochemical and plasma etching. Small 2010; 6(14):1469-73.

[15] Masubuchi S, Arai M, Machida T. Atomic force microscopy based tunable local anodic oxidation of graphene. Nano Lett 2011; 11 (11):4542-6.

[16] Puddy RK, Chua CJ, Buitelaar MR. Transport spectroscopy of a graphene quantum dot fabricated by atomic force microscope nanolithography. Appl Phys Lett 2013; 103(18): 183117.

[17] Arai M, Masubuchi S, Nose K, Mitsuda Y, Machida T. Fabrication of 10-nm-scale nanoconstrictions in graphene using atomic force microscopy-based local anodic oxidation lithography. Jpn J Appl Phys 2015; 54(4S):04DJ06.

[18] Lee DH, Kim CK, Lee JH, Chung HJ, Park BH. Fabricating in-plane transistor and memory using atomic force microscope lithography towards graphene system on chip. Carbon 2016; 96:223-8.

[19] Espinosa FM, Ryu YK, Marinov K, Dumcenco D, Kis A, Garcia R. Direct fabrication of thin layer $\mathrm{MoS}_{2}$ field-effect nanoscale transistors by oxidation scanning probe lithography. Appl Phys Lett 2015; 106(10):103503.

[20] Dago AI, Ryu YK, Garcia R. Sub-20 nm patterning of thin layer $\mathrm{WSe}_{2}$ by scanning probe lithography. Appl Phys Lett 2016; 109(16):163103.

[21] Lazzarino M, Heun S, Ressel B, Prince KC, Pingue P, Ascoli C. Atomic force microscope anodic oxidation studied by spectroscopic microscopy. Appl Phys Lett 2002; 81(15):2842.

[22] Tello M, Garcia R, Martin-Gago JA, Martínez NF, Martín-González MS, Aballe L, et al. Bottom-up fabrication of carbon-rich silicon carbide nanowires by manipulation of nanometer-sized ethanol menisci. Adv Mater 2005; 17(12):1480-3.

[23] Chien HM, Chuang MC, Tsai HC, Shiu HW, Chang LY, Chen CH, et al. On the nature of defects created on graphene by scanning probe lithography under ambient conditions. Carbon 2014; 80:318-24.

[24] Chuang MC, Chien HM, Chain YH, Chi GC, Lee SW and Woon WY. Local anodic oxidation kinetics of chemical vapor deposition graphene supported on a thin oxide buffered silicon template. Carbon 2013; 54: 336-42. 
[25] Lin YM, Dimitrakopoulos C, Jenkins KA, Farmer DB, Chiu HY, Grill A, et al. 100$\mathrm{GHz}$ transistors from wafer-scale epitaxial graphene. Science 2010; 327(5966):662.

[26] Schwierz F. Graphene transistors. Nat Nanotech 2010; 5(7):487-96.

[27] Dong R, Guo Z, Palmer J, Hu Y, Ruan M, Hankinson J, et al. Wafer bonding solution to epitaxial graphene-silicon integration. J Phys D Appl Phys 2014; 47(9):094001.

[28] Fan J, Michalik JM, Casado L, Roddaro S, Ibarra MR, De Teresa JM. Investigation of the influence on graphene by using electron-beam and photo-lithography. Solid State Commun 2011; 151(21):1574-8.

[29] Alaboson JMP, Wang QH, Kellar JA, Park J, Elam JW, Pellin MJ, et al. Conductive atomic force microscope nanopatterning of epitaxial graphene on $\mathrm{SiC}(0001)$ in ambient conditions. Adv Mater 2011; 23(19):2181-4.

[30] Rius G, Camara N, Godignon P, Pérez-Murano F, Mestres N. Nanostructuring of epitaxial graphene layers on $\mathrm{SiC}$ by means of field-induced atomic force microscopy modification. J Vac Sci Technol B 2009; 27(6):3149-52.

[31] Colangelo F, Piazza V, Coletti C, Rodaro S, Beltram F, Pingue P. Local anodic oxidation on hydrogen-intercalated graphene layers: oxide composition analysis and role of the silicon carbide substrate. Nanotechnology 2017; 28(10):105709.

[32] Ahn JJ, Jo YD, Kim SC, Lee JH, Koo SM. Crystallographic plane-orientation dependent atomic force microscopy-based local oxidation of silicon carbide. Nanoscale Res Lett 2011; $6(1): 235$.

[33] Bonaccorso F, Lombardo A, Hasan T, Sun Z, Colombo L, Ferrari AC. Production and processing of graphene and $2 \mathrm{~d}$ crystals. Mater Today 2012; 15(12):564-89.

[34] Tararan A, Zobelli A, Benito AM, Maser WK, Stéphan O. Revisiting graphene oxide chemistry via spatially-resolved electron energy loss spectroscopy. Chem Mater 2016; 28(11):3741-48.

[35] Mkhoyan KA, Contryman AW, Silcox J, Stewart DA, Eda G, Mattevi C, et al. Atomic and electronic structure of graphene-oxide. Nano Lett 2009; 9(3):1058-63.

[36] Egerton RF, Scattering delocalization and radiation damage in STEM-EELS. Ultramicroscopy 2017; 180: 115-124. 